\title{
Human mesenchymal stem cells enhance autophagy of lung carcinoma cells against apoptosis during serum deprivation
}

\author{
MING-HUI ZHANG, YI-DE HU, YU XU, YU XIAO, YAN LUO, ZONG-CHANG SONG and JING ZHOU \\ The Third Department of Oncology, PLA Cancer Research Institute of the Second Affiliated Hospital, \\ The Third Military Medical University, Chongqing 400037, P.R. China
}

Received November 22, 2012; Accepted January 22, 2013

DOI: $10.3892 /$ ijo.2013.1810

\begin{abstract}
Currently, some evidence suggests that human multipotential mesenchymal stems cells (hMSCs) aid tumor growth and metastasis. Nutrient deprivation and oxygen deficiency are representative characteristics of solid tumor microenvironment during the cancer development. Because the effects of hMSCs on tumors under stressful conditions have not been determined, we investigated the survival mechanisms used by stressed stromal cells on A549 and SPC-1 lung carcinoma cell lines in vitro and in vivo. An indirect culture system was used to investigate the effects of hMSCs on viability and apoptosis in starved carcinoma cells and focused on the role of autophagy in regulating the survival of carcinoma cells. The results showed that A549 and SPC-1 cells had higher viability when co-cultured with hMSCs and that this was mainly attributed to decreased apoptosis. Autophagosomes were analyzed using GFP-LC3 and electron microscopy, which showed that autophagy was significantly activated in the starved co-culture groups. However, the inhibition of autophagy by the autophagic inhibitor 3-MA significantly abrogated the apoptosis reduction in either single groups or co-culture groups under serum deprivation, which implied that the hMSCs protected against apoptosis by enhancing autophagy in lung carcinoma cells in vitro. We also observed that hMSCs promoted tumor initiation and growth in vivo. In conclusion, our study demonstrates that hMSCs can protect carcinoma cells from nutrient deprivationinduced apoptosis and promote tumor initiation and growth, and, interestingly, autophagy plays an important role in the survival of cancer cells.
\end{abstract}

Correspondence to: Dr Yi-De Hu, The Third Department of Oncology, PLA Cancer Research Institute of the Second Affiliated Hospital, The Third Military Medical University, Chongqing 400037, P.R. China

E-mail: huyide_mit@yahoo.com.cn

Key words: hMSCs, lung carcinoma cells, apoptosis, autophagy, serum deprivation

\section{Introduction}

Currently, lung cancer is the leading cause of cancer-related mortality throughout the world (1). Although there have been significant advances in cancer treatments, this malignancy remains poorly responsive to conventional therapy. Hence, it is urgent to determine the survival mechanism of carcinoma cells to develop more efficient therapies for patients.

Human mesenchymal stem cells (hMSCs) are pluripotent progenitor cells that reside within the adult bone marrow. They have self-renewal capacity, long-term viability and can differentiate into the adipocytic, chondrocytic or osteocytic lineages (2-4). Although hMSCs reside predominantly in the bone marrow, they are also distributed throughout many other tissues, where they are thought to function as local sources of dormant stem cells (5). After injury or chronic inflammation, the wounded tissue would release specific endocrine signals that are then transmitted to the bone marrow, leading to the mobilization of multi-potent hMSCs and their subsequent recruitment to the damage site (6). Moreover, recent evidence has indicated that hMSCs are recruited and incorporated within the connective tissue stroma of tumors (7-10). In the tumor microenvironment, hMSCs can secrete several tumor growth-promoting factors, favoring tumor growth, enhancing tumor vessel formation, promoting cancer metastasis and creating tumor stem cell niches (11-14). The ability of hMSCs to home to sites of injury and tumors has encouraged investigation of these cells as potential therapeutic tools.

Nutrient deprivation and oxygen deficiency are representative characteristics of the solid tumor microenvironment during cancer development $(15,16)$. Autophagy is a wellestablished mechanism for degrading cytoplasmic proteins, macromolecules, and organelles to provide a nutrient source to promote the survival of cells that are under metabolic stress $(17,18)$. Starvation increases the number and size of autophagosomes in many tissues, suggesting that autophagy is a critical component of the body's response to nutrient deprivation and amino acid/fuel homeostasis. Autophagy has been implicated in a number of different physiological and pathological conditions, including development, differentiation, immunity, aging and cell death $(19,20)$. In addition, accumulating evidence demonstrates interesting links between 
autophagy and tumorigenesis, tumor progress and chemoresistance $(21,22)$. In particular, the regulation of autophagy in carcinoma cells is complex because it can enhance tumor cell survival in response to certain stresses.

Because stromal cells play an important role in solid tumor development, there is a significant gap in our understanding of the relationship between stromal cells and carcinoma cells under stressful conditions, such as hypoxia or nutrient deprivation. In this study, we investigated the influence of hMSCs on A549 and SPC-1 cells under nutrient deprivation and the role of autophagy in this context, in lung carcinoma cells. Our study demonstrates that hMSCs can protect carcinoma cells from nutrient deprivation-induced apoptosis, and interestingly, autophagy plays an important role in this protection. Further, we found that hMSCs promoted earlier tumor initiation and growth in vivo. This result indicated that protection by stromal cells is a factor that helps lung carcinoma cells survive and proliferate continually in the ischemic microenvironment, even under extreme nutrient limitation.

\section{Materials and methods}

Cell culture and reagents. The human lung carcinoma cell lines A549 and SPC-1 were purchased from the American Type Culture Collection (Manassas, VA) and maintained in Dulbecco's modified Eagle's medium: Nutrient Mixture F-12 (DMEM/F12) (Gibco, Invitrogen), containing 10\% fetal bovine serum (FBS) (Gibco, Invitrogen), $100 \mathrm{U} / \mathrm{ml}$ penicillin and $100 \mu \mathrm{g} / \mathrm{ml}$ streptomycin in a humidified incubator under $95 \%$ air and $5 \% \mathrm{CO}_{2}$ at $37^{\circ} \mathrm{C}$. 3-MA and DMSO were purchased from Sigma-Aldrich. Both DMSO and 3-MA were used at $5 \mathrm{mM}$.

HMSCs were isolated from hip aspirates of two male healthy donors with locally approved informed consent. The marrow was diluted twice with phosphate buffered saline (PBS) and then isolated by Percoll (Sigma-Aldrich) density-gradient centrifugation (specific gravity 1.073). Primary cells were collected and incubated in DMEM/F12 containing 10\% FBS, $0.2 \mathrm{mmol} / 1$ glutamine, $100 \mathrm{U} / \mathrm{ml}$ penicillin and $100 \mu \mathrm{g} / \mathrm{ml}$ streptomycin under $95 \%$ air and $5 \% \mathrm{CO}_{2}$ at $37^{\circ} \mathrm{C}$. After $48 \mathrm{~h}$, the medium was replaced and non-adherent cells were discarded. After 3-5 passages, the cells met the minimal criteria for defining multipotent mesenchymal stromal cells with typical CD45-, CD34-, CD14-, CD19-, HLA-DR-, CD73+, CD90+ and CD105+ expression (23), as identified by flow cytometry (data not shown). Passages three to five hMSCs were used in this study.

Cell co-culture or hMSC SD-conditioned medium treatment. Co-culture systems were established using 6-well transwell (0.4 $\mu \mathrm{m}$ pore, Corning) plates. hMSCs were plated at $1 \times 10^{5}$ cells per insert and A549 or SPC-1 cell suspensions $2 \times 10^{5}$ cells per well were placed in the lower compartment of the culture well. The ratio of carcinoma cells to hMSCs (2:1 ratio) was selected according to previously optimized conditions for high tumor implantation rate (24). After $6 \mathrm{~h}$, both the inserts and lower culture wells were washed three times with PBS and then the medium was switched to DF-12 without FBS for $24 \mathrm{~h}$.

Serum-deprived hMSC conditioned medium (hMSC SD-conditioned medium) were also used as indirect co-culture medium. hMSCs were cultured until they reached $80 \%$ confluence, washed three times with PBS and incubated in DF-12 medium without FBS for $24 \mathrm{~h}$. Then, SD-conditioned media were harvested by $0.22 \mu \mathrm{m}$ filtration.

Serum-deprived co-culture groups (SD+co-culture) grew in the presence of hMSCs in DF-12 media without FBS for $24 \mathrm{~h}$; SD-conditioned medium groups (SD-conditioned) were grown in the presence of only hMSCs SD-conditioned media for $24 \mathrm{~h}$; serum-deprived control groups (SD+control) were grown in DF-12 media without FBS; and control groups (control) were grown in DF-12 media with FBS for $24 \mathrm{~h}$. Every group had three wells.

Cell Counting Kit-8. The measurement of viable cell mass was performed with a Cell Counting Kit-8 (CCK8, Beyotime, Jiangsu, China) assay. Cells were first seeded in 24-well flatbottomed plates for $6 \mathrm{~h}$. Next, the wells were prepared for the four different culture groups, and the cells were grown for $24 \mathrm{~h}$. Cell proliferation was measured according to the manufacturer's instructions.

Cell apoptosis assay. Apoptotic cells were analyzed by flow cytometry. Four groups were cultured in different culture media for $24 \mathrm{~h}$. Approximately $2 \times 10^{5}$ cells were incubated with the Cell Apoptosis Assay according to the manufacturer's instructions (Beyotime). Briefly, cells were resuspended in $195 \mu \mathrm{l} 1 \mathrm{X}$ binding buffer containing $5 \mu \mathrm{l}$ Annexin $\mathrm{V}$ for $10 \mathrm{~min}$ at room temperature in the dark, and then mixed with another $190 \mu \mathrm{l} 1 \mathrm{X}$ binding buffer containing $10 \mu \mathrm{l} \mathrm{PI}$ and incubated in an ice bath for another $10 \mathrm{~min}$. After incubation, at least 10,000 cells were measured on a Beckman Coulter flow cytometer. Cells undergoing an early stage apoptosis are stained with Annexin V-FITC only, while cells at a late stage of apoptosis and necrotic cells are stained with both Annexin V-FITC and propidium iodide.

Transfection. GFP-tagged microtubule-associated protein 1 light chain 3 (GFP-MAP1LC3) was used to monitor autophagy through direct fluorescence microscopy. Cells undergoing autophagy were observed to have significant numbers of punctate GFP, while normal cells showed a primarily diffuse GFP signal. The carcinoma cells were seeded $\left(2 \times 10^{5}\right.$ cells/ well) in 6-well plates overnight. The cells were transiently transfected using Lipofectamine 2000 Plus GFP-MAP1LC3 (Yrgene, China) according to the manufacturer's protocol. The cells were cultured for $24 \mathrm{~h}$ to ensure the expression of GFP-MAP1LC3, divided into the four experimental groups, and incubated for $24 \mathrm{~h}$. Then, the cells were fixed in $4 \%$ paraformaldehyde for $10 \mathrm{~min}$, stained with DAPI and analyzed under a Leica laser confocal microscope to measure the cells with GFP-MAP1LC3-positive dots.

Transmission electron microscopy (TEM). After treatment with different culture media for $24 \mathrm{~h}$, cells were fixed in ice-cold $2.5 \%$ glutaraldehyde acid in $0.1 \mathrm{M}$ PBS buffer for $2 \mathrm{~h}$ or longer, rinsed with PBS, postfixed in $1 \%$ osmium tetroxide with $0.1 \%$ potassium ferricyanide, dehydrated in a graded series of ethanol (30-90\%) and embedded in Epon resin. Thin sections of 50-60 nm were cut and picked up on copper grids, post-stained with uranyl acetate and lead citrate and then observed using a Philips EM420 transmission electron microscopy. 
Western blot analysis. At the end of the treatments, cells were lysed in cell lysis buffer (Beyotime) with $1 \mathrm{mM}$ phenylmethylsulfonyl fluoride (PMSF), and the protein concentration of the lysate was quantified using a BCA protein assay kit (Beyotime). Equal amounts of protein for each sample were electrophoresed in SDS-PAGE and transferred to a polyvinylidene fluoride (PVDF) membrane. After blocking with $5 \%$ non-fat milk, the membranes were incubated with primary antibodies against MAP1LC3, Beclin-1, B-cell lymphoma/leukemia-2 (Bcl-2) (Abcam Inc.) and $\beta$-actin (Beyotime) overnight at $4^{\circ} \mathrm{C}$. Then, the membranes were incubated with a horseradish peroxidase-conjugated goat anti-rabbit IgG secondary antibody (Beyotime) for $2 \mathrm{~h}$. Bands were detected using ECL (Beyotime). Protein levels were quantitated by densitometry using Quantity One software (Bio-Rad Laboratories, Munich, Germany).

Animal studies. Male NOD/SCID mice aged 4-6 weeks were used in our study. All study protocols were approved by the Third Military Medical University Animal Care and Use Committee (Chongqing, China; no. 2011-11). All procedures were carried out in accordance with the advice and permission of the Institutional Ethics Committee. Two lines of carcinoma cells were treated with or without hMSCs for $24 \mathrm{~h}$ under serum-deprived medium and then resuspended as single-cell type suspensions $\left(1 \times 10^{6}\right)$ or mixed with hMSCs $\left(5 \times 10^{5}\right)$ in $0.1 \mathrm{ml}$ PBS. Cells were injected subcutaneously into the left flank of the mice. The mice were examined each week and palpable tumors at the injection sites with a size of more than $3 \mathrm{~mm}$ in diameters were considered tumors. After 30 days, the mice were sacrificed and tumor volume was calculated according to the formula $\mathrm{V}=\pi / 6$ (length $\mathrm{x}$ width $\mathrm{x}$ height). Each group included $10 \mathrm{NOD} / \mathrm{SCID}$ mice.

Statistical analysis. All experiments were repeated at least three times. The statistical analyses were performed using SPSS v.13.0. The data were expressed as the means \pm SD and analyzed using the Student's t-test. The criterion for statistical significance was taken as $\mathrm{p}<0.05$.

\section{Results}

Lung carcinoma cells have better tolerance with co-culture or hMSCs SD-conditioned media in serum-deprived conditions. There is evidence showing that hMSCs provide sufficient stromal support for tumor cells. Solid tumors are typically characterized by nutrient deprivation and an ischemic microenvironment as they grow. To determine whether hMSCs influence lung carcinoma cell survival, the lung cancer cell lines A549 and SPC-1 were used. The two cell lines were cultured in DMEM/F12 with or without hMSCs under serum-deprived conditions or with hMSC SD-conditioned media for $24 \mathrm{~h}$. At the end of the treatment, cell viability analysis showed that hMSCs helped maintain the viability of A549 and SPC-1 under serum deprived conditions. The viability levels of A549 and SPC-1 cells in the SD+co-culture groups were higher than those in the $\mathrm{SD}+\mathrm{control}$ groups $(\mathrm{p}<0.01)$ and similar to those of the SD-conditioned groups $(p<0.01)$. Moreover, the viability of SD+co-culture was similar to that of the SD-conditioned groups (Fig. 1).
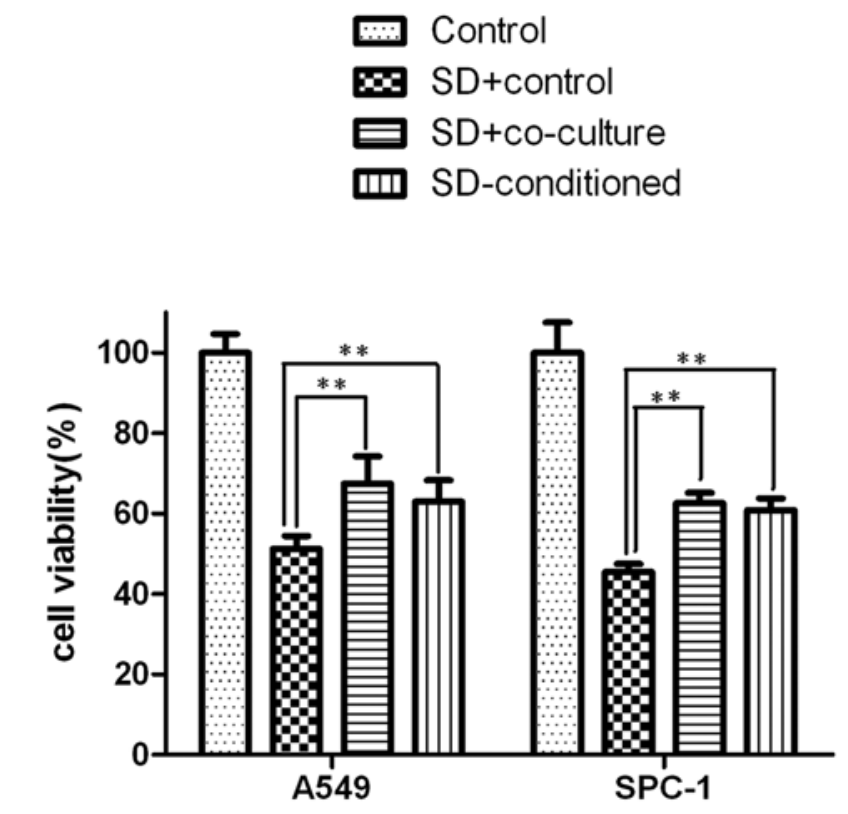

Figure 1. hMSCs protect the proliferation capacity and viability of lung carcinoma cells. The lung carcinoma cell lines A549 and SPC-1 were co-cultured with hMSCs using transwell co-culture systems (SD+co-culture) or treated with hMSC SD-conditioned medium (SD-conditioned) for $24 \mathrm{~h}$ under serumdeprived conditions. Serum-deprived control groups (SD+control) were grown in DF-12 media without FBS and control cells (Control) were grown in DF-12 media with FBS for $24 \mathrm{~h}$. At the end of treatment, cell proliferation and viability were measured using the cell counting kit- 8 assay. The cell viability is shown as the mean \pm SD. ${ }^{*} \mathrm{p}<0.05,{ }^{* *} \mathrm{p}<0.01$.

The survival of lung carcinoma cells is attributed to the decrease in apoptosis induced by hMSCs under serum deprivation. As higher viability was observed for lung carcinoma cells in the co-cultured and SD-conditioned groups, we investigated the effects of hMSCs on the apoptosis of tumor cells, which occurs in stressful conditions. The Annexin V/PI assay was used to detect apoptosis by flow cytometry. Our findings showed that the apoptotic rate of A549 and SPC-1 cells was obviously reduced in the $\mathrm{SD}+\mathrm{co}$-culture and $\mathrm{SD}$-conditioned groups compared with $\mathrm{SD}+$ control $(\mathrm{p}<0.01)$. Likewise, the apoptotic rate of A549 and SPC-1 SD+co-culture resembled that of the SD-conditioned groups (Fig. 2) ( $>>0.05)$.

Autophagy is activated by hMSCs in lung carcinoma cells under serum deprivation conditions. To determine whether autophagy is involved in the hMSC-mediated increase in lung carcinoma cell survival upon nutrient deprivation, we examined the accumulation of autophagosomes (25). After transient transfection with GFP-MAP1LC3 plasmids, A549 and SPC-1 cells were incubated in the four different experimental media for $24 \mathrm{~h}$, and we then observed GFP-MAP1LC3 dots under a fluorescence microscope (Fig. 3A). Transmission electron microscopy was used to examine autophagic vacuoles (Fig. 3B). The A549 and SPC-1 cells in the SD+co-culture and SD-conditioned groups obviously have more autophagosomes than SD+control, and the SD+co-culture resembled the SD-conditioned groups (Fig. 3C). We also evaluated MAP1LC3-I and MAP1LC3-II protein levels using western blot analysis (Fig. 3D). 

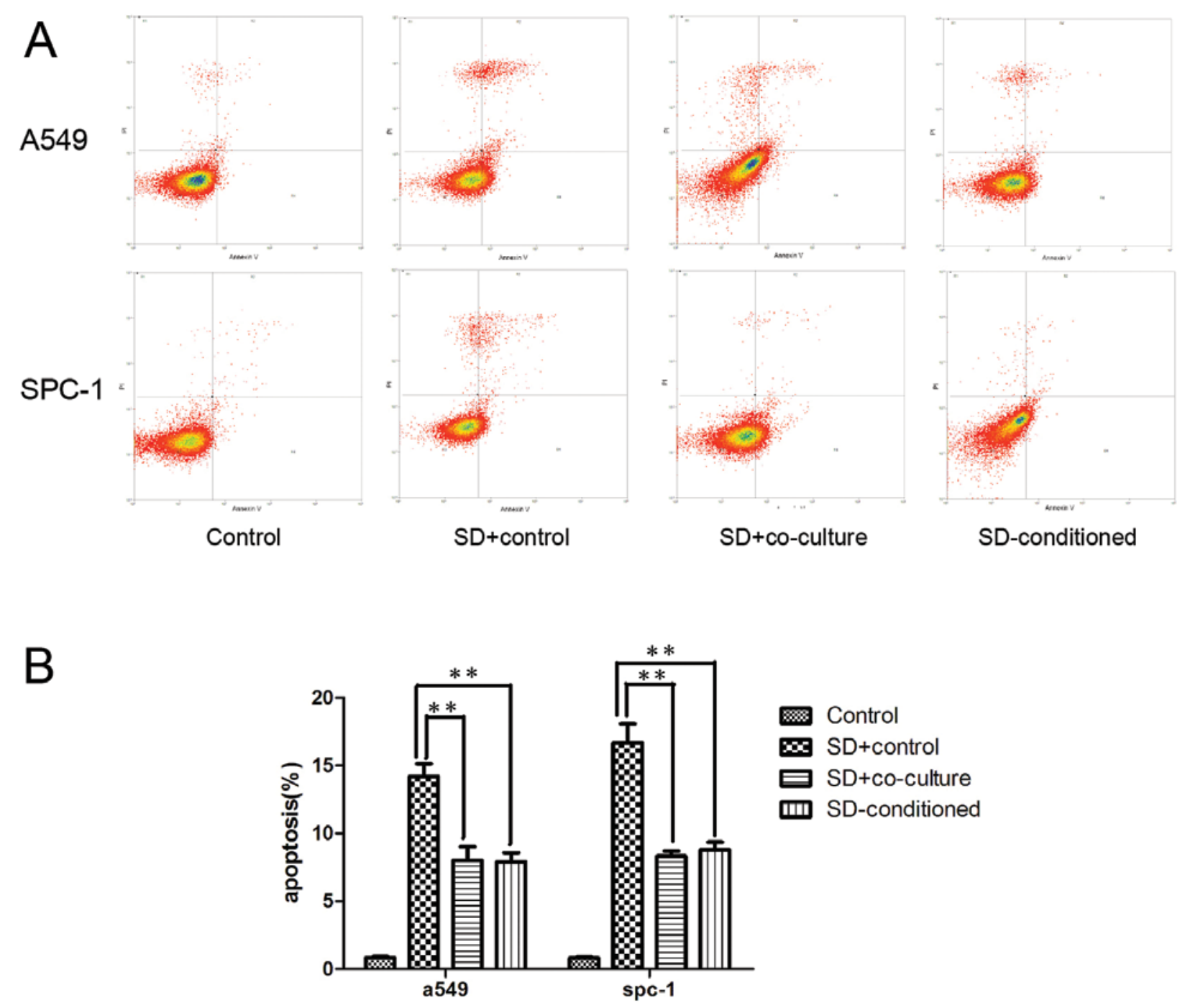

Figure 2. Apoptosis of lung carcinoma cells was decreased by hMSCs under serum deprivation. (A) A549 and SPC-1s were treated as detailed in Fig. 1. Apoptosis was detected by flow cytometry using the Annexin V/PI assay. (B) The apoptotic rates of A549 and SPC-1 in the four groups were quantified. Mean data $( \pm \mathrm{SD})$ are shown. ${ }^{*} \mathrm{p}<0.05,{ }^{* *} \mathrm{p}<0.01$.

Autophagy activated by hMSCs is involved in the tolerance of lung carcinoma cells to serum deprivation. To analyze whether autophagy is involved in the observed decrease in apoptosis, the effect of autophagy inhibition on cell viability was observed. The autophagy inhibitor 3-methyladenine (3-MA) (26) was used to block autophagy. SD+control group and SD-conditioned group were treated with DMSO or 3-MA, respectively. A549 and SPC-1 cells incubated with or without hMSC in serum deprivation lacked autophagic activity when treated with 3-MA (Fig. 4A and B). Moreover, greater numbers of apoptotic cells were observed in the 3-MA treatment groups compared to the non-3-MA-treated groups (Fig. 4C and D).

hMSCs favor tumorigenesis and growth after starvation in vivo. To investigate the effects of hMSCs on the growth of A549 cells and SPC-1 cells after starvation in vivo, we assessed the growth of tumor cells in NOD/SCID mice. After 2 weeks, all mice transplanted with carcinoma cells and hMSCs have palpable tumor nodules, compared to only 70 and $60 \%$ of the mice injected with carcinoma cells alone (Fig. 5A). The mean volume of tumors of the mice co-injected with hMSCs and tumor cells was dramatically larger than that of the control groups (Fig. 5B).

Protein expression changes in the tumor cells induced by the $S D$-hMSC-conditioned medium. To elucidate the molecular mechanism underlying the protective effects of hMSCs on tumor cells in vitro, we investigated the expression of Beclin-1 and $\mathrm{Bcl}-2$ in tumor cells by western blot analysis. The results showed that the presence of MSCs during serum deprivation increased the levels of Beclin-1, a mammalian autophagy protein, in A549 and SPC-1 cells. In contrast, the expression of Bcl-2, an anti-apoptotic and anti-autophagy protein, was clearly reduced in the SD-conditioned groups. Both Beclin-1 and Bcl-2 had statistically significant differences in expression in the $\mathrm{SD}$-conditioned groups and $\mathrm{SD}+$ control groups (Fig. 6A and B). 
A

A549
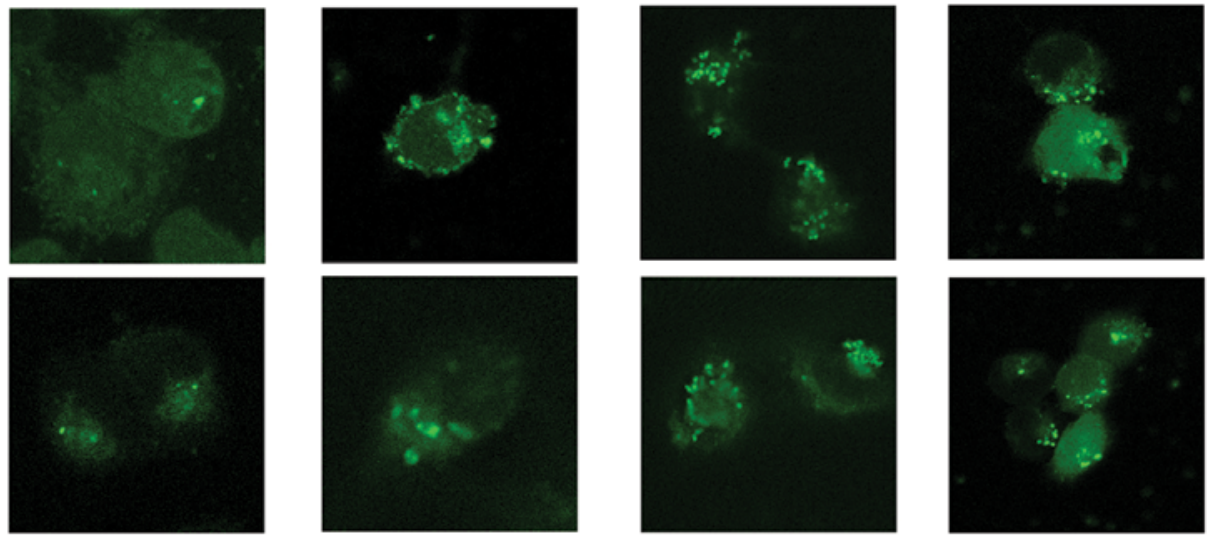

B
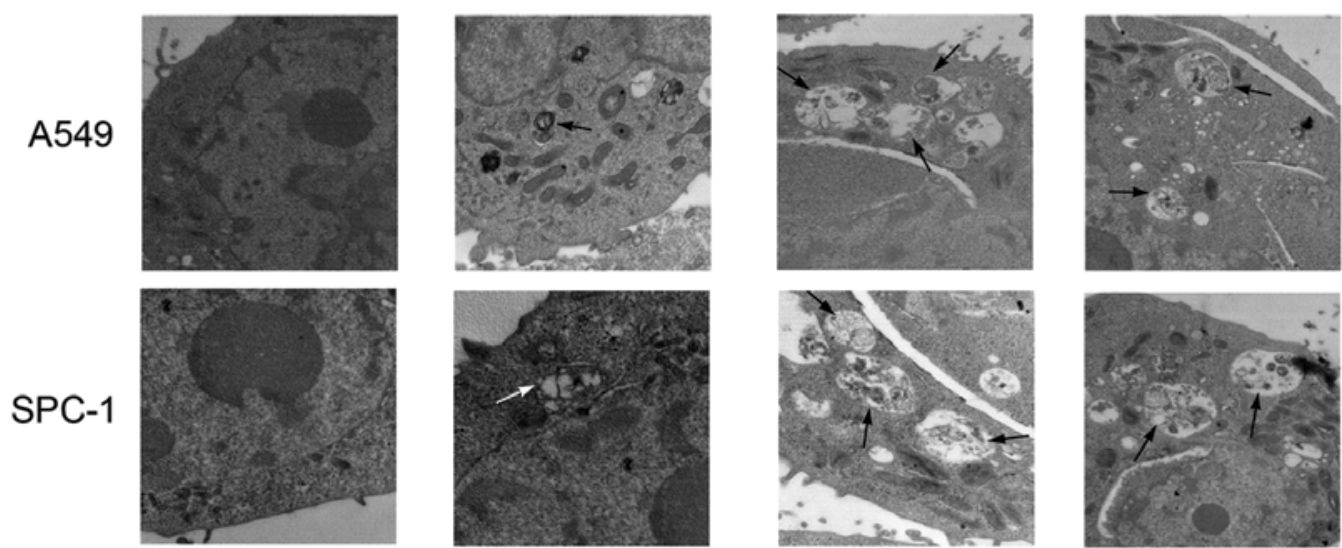

SD+control

SD+co-culture

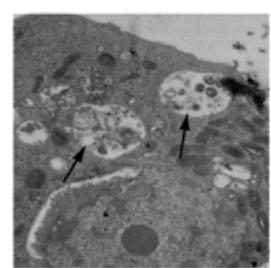

SD+conditioned

C

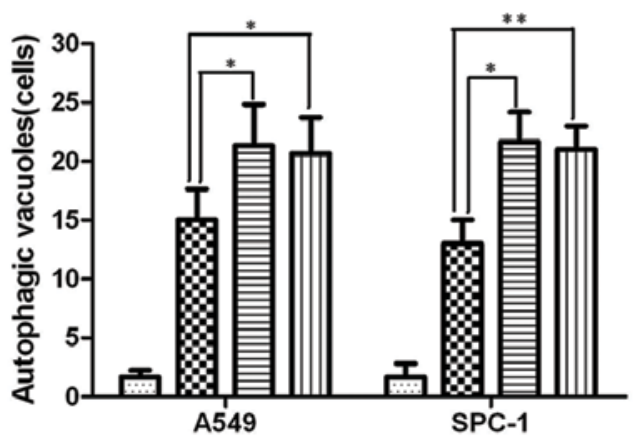

四 Control

S SD+control

$\boxminus$ SD+co-culture

III SD-conditioned

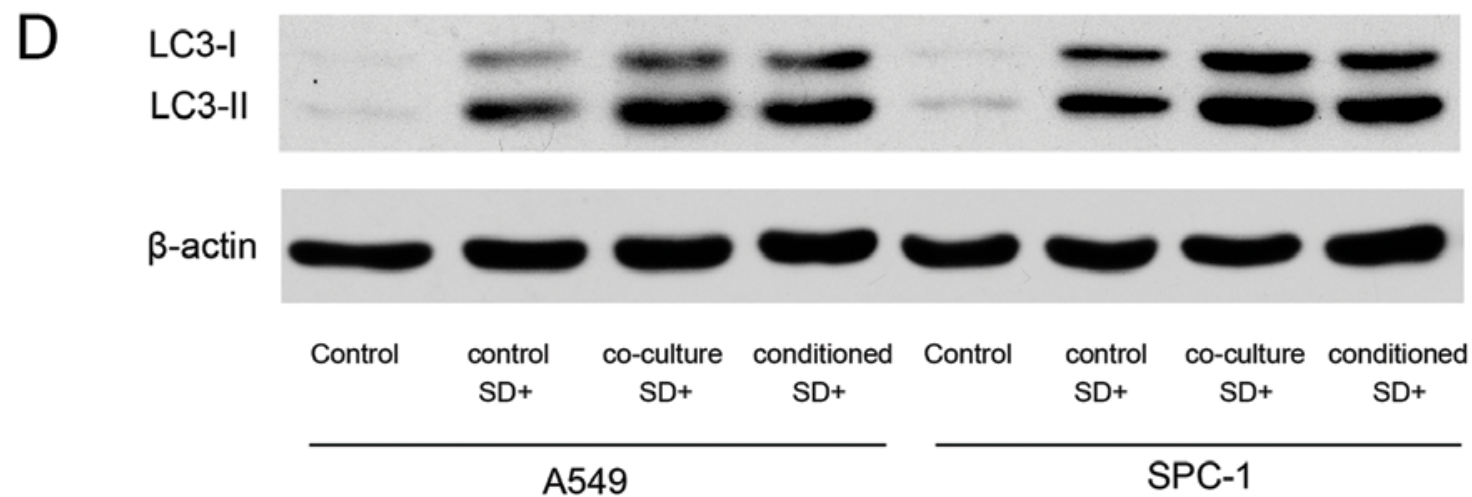

Figure 3. hMSC activated autophagy in lung carcinoma cells under nutrient deprivation. (A) After transient transfection with GFP-MAP1LC3 plasmids, A549 and SPC-1 cells were treated as indicated in Fig. 1, and GFP-MAP1LC3 dots were observed under a fluorescence microscope. (B) Representative electron microscopic images showing autophagic vacuoles (arrows). (C) The number of cells with autophagic vacuoles was quantified in 30 cells per group. ${ }^{*} \mathrm{p}<0.05,{ }^{* *} \mathrm{p}<0.01$. (D) Expression of MAP1LC3-I and MAP1LC3-II as determined by western blot analysis. 
A

A549
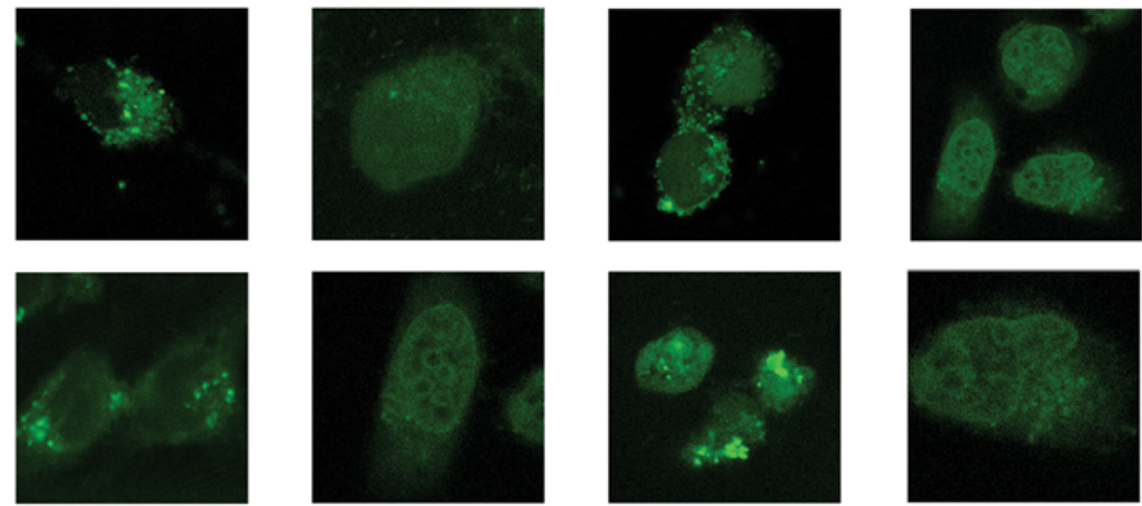

hMSC -

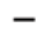

$+$

3MA -

$+$

$+$

$+$

B

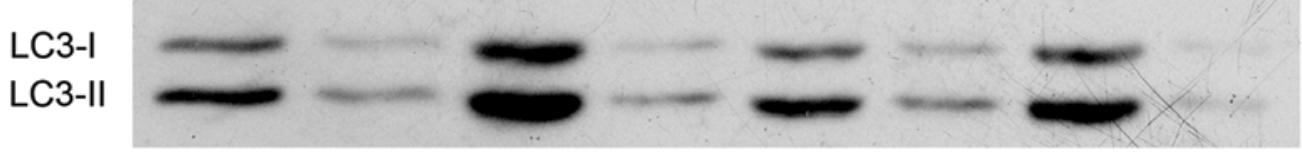

$\beta$-actin
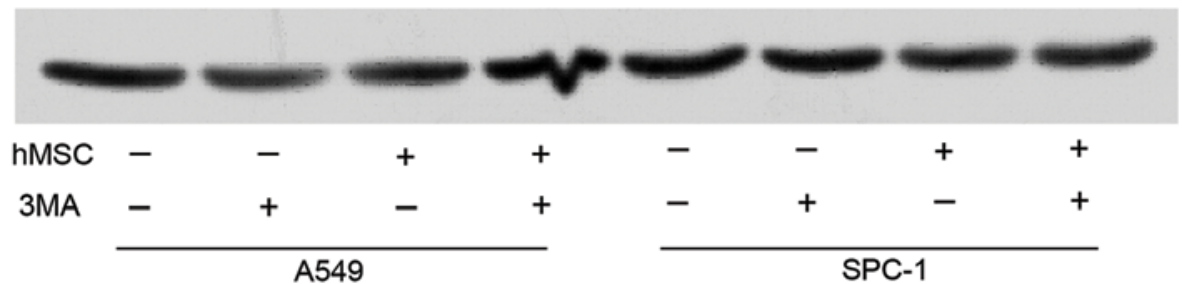

C
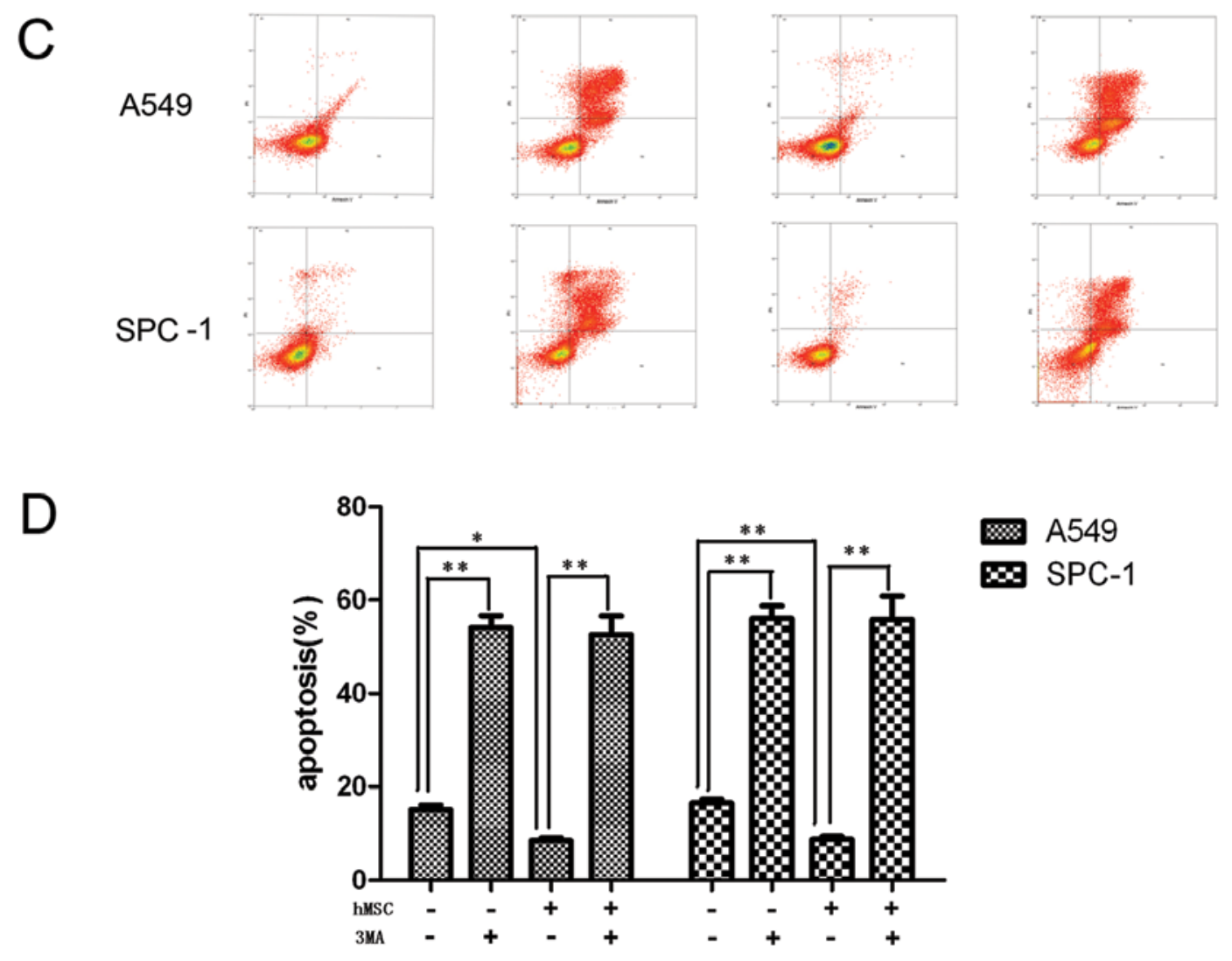

A549

$\infty$ SPC-1

Figure 4. Autophagy activated by hMSC is involved in the survival of lung carcinoma cells under serum deprivation. (A) The SD+control group and SD-conditioned group were treated with DMSO or 3-MA and GFP-MAP1LC3 was observed under a fluorescence microscope. (B) The expression of MAP1LC3-I and MAP1LC3-II, as measured by western blot analysis. (C) Apoptosis was detected by Annexin V/PI staining followed by flow cytometry. (D) The apoptotic rates of the SD+control group and SD-conditioned group treated with DMSO or 3-MA were quantified. The means $( \pm \mathrm{SD})$ are shown. ${ }^{*} \mathrm{p}<0.05$, ${ }^{* *} \mathrm{p}<0.01$. 

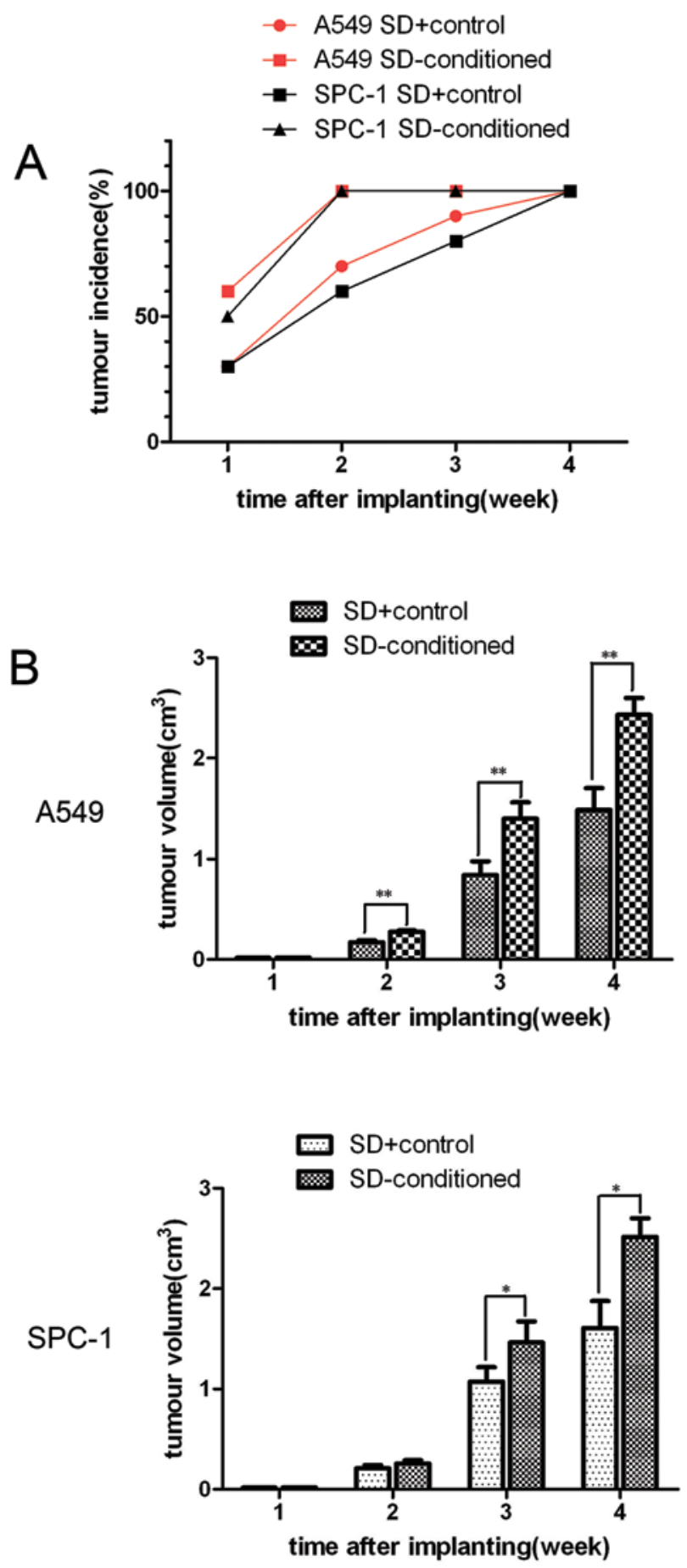

Figure 5. MSCs favor tumorigenesis and growth after starvation in vivo. (A) Tumorigenesis in mice co-injected with tumor cells and hMSCs or control mice injected with tumor cells alone. (B) The mean tumor volumes of the co-injected and control groups. ${ }^{*} \mathrm{p}<0.05,{ }^{* *} \mathrm{p}<0.01$.

\section{Discussion}

Solid tumors are composed of tumor cells and supportive nontumor components known as tumor stroma. There is a niche that is enriched with carcinoma cells and a subpopulation of closely associated stromal cells that control the activity of the carcinoma cells. hMSCs can be recruited to these niches and subsequently secrete various cytokines and growth
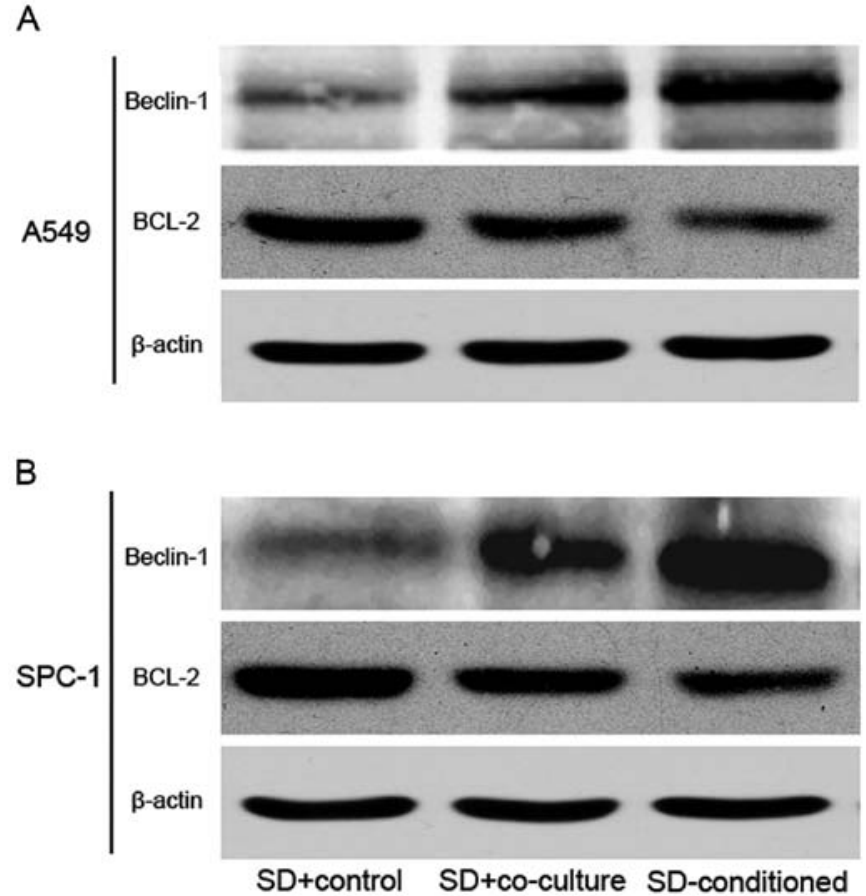

Figure 6. Protein expression changes in tumor cells induced by SD-hMSC-conditioned medium. (A) The expression of Beclin-1 and Bcl-2 in control, SD+control and SD-conditioned A549 cells. (B) The expression of Beclin-1 and Bcl-2 in control, SD+control and SD-conditioned SPC-1 cells.

factors (7). When tumors grow beyond $2 \mathrm{~mm}$ in diameter, carcinoma cells and stromal cells could undergo starvation due to lack of sufficient nutrients in the niche (27). Because of the close relationship between hMSCs and carcinoma cells, it is unclear how hMSCs influence the fate of carcinoma cells under nutrient deprivation conditions. In the present study, we used an SD co-culture system and SD-hMSC-conditioned medium to explore the effects of hMSCs on lung carcinoma cell lines A549 and SPC-1. This is the first time the role of hMSCs in lung carcinoma cells under serum deprivation has been addressed. We found that the viability of carcinoma cells grown with hMSCs is higher than that of cells grown without hMSCs after serum starvation for $24 \mathrm{~h}$. Moreover, a marked decrease in apoptosis was observed in lung carcinoma cells that were co-cultured with hMSCs, supporting the protective role of hMSCs against apoptosis.

Autophagy is a well-established mechanism to degrade cytoplasmic proteins, macromolecules, and organelles and to provide a nutrient source to promote the survival of cells in metabolic distress. Accumulating evidence supports a role for autophagy in maintaining tumor cell survival in response to metabolic stress and hypoxia (28-31). Autophagy has been considered an indispensable physiological reaction for sustaining cell viability during starvation. A549 and SPC-1 cells were co-cultured with hMSCs during nutrient deprivation showed more autophagic cells than were observed in SD-condition groups. Less apoptosis was observed in the co-culture system. After the addition of 3-MA, a commonly used inhibitor of starvation or rapamycin-induced autophagy, apoptosis increased, concomitant with a decrease in 
autophagy. Moreover, cells at late stage apoptosis and necrotic cells were found in the 3-MA groups, supporting the protective role of hMSCs against apoptosis under nutrient starvation conditions. Similarly, 3-MA treatment also led to a dramatic decrease in the survival of single culture group under starvation conditions. This is similar to findings in starved HeLa cells, in which it was shown that the suppression of autophagy could promote apoptosis and caspase-3 activation (32). The indirect system used in this study indicated that this protective mechanism may involve cytokines such as growth factors, anti-apoptotic factors and TGF- $\beta$, which have been found in conditioned medium from SD-hMSCs (27).

In vivo, subcutaneous injection experiments showed that starved lung carcinoma cells that were co-injected with hMSCs exhibited stronger tumor initiation and growth than carcinoma cells that were injected alone. There are several possible explanations for this observation. First, tumor initiation and growth may be promoted by the protective role of hMSCs on lung carcinoma cell viability and apoptosis, as reported in this study. Further, there is evidence that hMSCs may differentiate into tumor stromal fibroblasts, forming a niche that favors tumor growth (33). Furthermore, hMSCs enhanced vascular endothelial growth factor (VEGF) expression in tumor cells (34). Importantly, as the tumor grows, the stromal cells and tumor could undergo starvation and stromal cells may secrete growth factors and anti-apoptotic factors that protect carcinoma cells (27). Overall, we hypothesize that SD-hMSCs may provide a protective niche for lung carcinoma cells. During tumor growth, the growth factors and anti-apoptotic factors secreted by hMSCs protect lung carcinoma cells and allow them to survive the nutrient deficient situation.

Beclin-1 is identified as mammalian autophagy gene that plays a major role in the formation of the autophagosome (35). It also interacts with anti-apoptotic multidomain proteins $\mathrm{Bcl}-2$ family members, in particular Bcl-2 and its homologue Bcl-XL, whose overexpression would inhibit the autophagy-inducing activity of Beclin-1 (36-38). Blocking the interaction between Beclin-1 and Bcl-2 has been reported to enhance autophagy (39). We determined that the expression levels of Beclin-1 and Bcl-2 proteins in A549 and SPC-1 cells after inoculation. Under normal conditions, A549 and SPC-1 cells exhibited a basal expression of Beclin-1 and Bcl-2; under serum deprivation conditions, the expression of $\mathrm{Bcl}-2$ was decreased and the expression of Beclin-1 increased. The addition of hMSCs resulted in a dramatic decrease in the levels of Bcl-2 and an increase in Beclin-1, which triggers autophagy as protective mechanism. Hence, we hypothesize that hMSCs could trigger autophagy by Beclin-1 without the inhibition of Bcl-2.

According to our studies, we conclude that hMSCs could promote tumor cell proliferation and reduce tumor cell apoptosis and that autophagy plays an important role in the survival of carcinoma cells. In vivo, earlier tumor initiation and growth were observed when tumor cells were subcutaneously injected along with hMSCs. The disruption of the Bcl-2 and Beclin-1 interaction may be involved in this autophagy protective mechanism. Taken together, this study provides preliminary exploratory research results for understanding stromal protection in the survival mechanism of lung carci- noma cells and show that the promotion of autophagy by hMSCs play an important role in tumor cell survival.

\section{References}

1. Siegel R, Desantis C, Virgo K, et al: Cancer treatment and survivorship statistics, 2012. CA Cancer J Clin 62: 220-241, 2012.

2. Prockop DJ: Marrow stromal cells as stem cells for nonhematopoietic tissues. Science 276: 71-74, 1997.

3. Young HE, Steele TA, Bray RA, et al: Human reserve pluripotent mesenchymal stem cells are present in the connective tissues of skeletal muscle and dermis derived from fetal, adult, and geriatric donors. Anat Rec (Hoboken) 264: 51-62, 2001.

4. Pittenger MF, Mackay AM, Beck SC, et al: Multilineage potential of adult human mesenchymal stem cells. Science 284 : 143-147, 1999.

5. Young HE, Duplaa C, Young TM, et al: Clonogenic analysis reveals reserve stem cells in postnatal mammals: I. Pluripotent mesenchymal stem cells. Anat Rec (Hoboken) 263: 350-360, 2001.

6. Fox JM, Chamberlain G, Ashton BA and Middleton J: Recent advances into the understanding of mesenchymal stem cell trafficking. Br J Haematol 137: 491-502, 2007.

7. Karnoub AE, Dash AB, Vo AP, et al: Mesenchymal stem cells within tumour stroma promote breast cancer metastasis. Nature 449: 557-563, 2007.

8. Mishra PJ,Humeniuk R,Medina DJ, et al: Carcinoma-associated fibroblast-like differentiation of human mesenchymal stem cells. Cancer Res 68: 4331-4339, 2008.

9. Studeny M, Marini FC, Champlin RE, Zompetta C, Fidler IJ and Andreeff M: Bone marrow-derived mesenchymal stem cells as vehicles for interferon-beta delivery into tumors. Cancer Res 62: 3603-3608, 2002.

10. Xin H, Kanehira M, Mizuguchi $\mathrm{H}$, et al: Targeted delivery of CX3CL1 to multiple lung tumors by mesenchymal stem cells. Stem Cells 25: 1618-1626, 2007.

11. Stagg J: Mesenchymal stem cells in cancer. Stem Cell Rev 4: 119-124, 2008.

12. Zhang W: Mesenchymal stem cells in cancer: friends or foes. Cancer Biol Ther 7: 252-254, 2008.

13. Bian ZY, Fan QM, Li G, Xu WT and Tang TT: Human mesenchymal stem cells promote growth of osteosarcoma: involvement of interleukin-6 in the interaction between human mesenchymal stem cells and Saos-2. Cancer Sci 101: 2554-2560, 2010.

14. Bagley RG, Weber W, Rouleau C, et al: Human mesenchymal stem cells from bone marrow express tumor endothelial and stromal markers. Int J Oncol 34: 619-627, 2009.

15. Kato K, Ogura T, Kishimoto A, et al: Critical roles of AMP-activated protein kinase in constitutive tolerance of cancer cells to nutrient deprivation and tumor formation. Oncogene 21: 6082-6090, 2002.

16. Li Y, Iglehart JD, Richardson AL and Wang ZC: The amplified cancer gene LAPTM4B promotes tumor growth and tolerance to stress through the induction of autophagy. Autophagy 8: 273-274, 2012.

17. Kelekar A: Introduction to the review series Autophagy in Higher Eukaryotes--a matter of survival or death. Autophagy 4: 555-556, 2008.

18. Yin L, Kharbanda S and Kufe D: MUC1 oncoprotein promotes autophagy in a survival response to glucose deprivation. Int $\mathrm{J}$ Oncol 34: 1691-1699, 2009.

19. Carew JS, Medina EC, Esquivel JA II, et al: Autophagy inhibition enhances vorinostat-induced apoptosis via ubiquitinated protein accumulation. J Cell Mol Med 14: 2448-2459, 2010.

20. Kang C, You YJ and Avery L: Dual roles of autophagy in the survival of Caenorhabditis elegans during starvation. Genes Dev 21: 2161-2171, 2007.

21. Mathew R, Karantza-Wadsworth V and White E: Role of autophagy in cancer. Nature reviews. Cancer 7: 961-967, 2007.

22. Fujii S, Mitsunaga S, Yamazaki M, et al: Autophagy is activated in pancreatic cancer cells and correlates with poor patient outcome. Cancer Sci 99: 1813-1819, 2008.

23. Dominici M, Le Blanc K, Mueller I, et al: Minimal criteria for defining multipotent mesenchymal stromal cells. The International Society for Cellular Therapy position statement. Cytotherapy 8: 315-317, 2006. 
24. Rhodes LV, Muir SE, Elliott S, et al: Adult human mesenchymal stem cells enhance breast tumorigenesis and promote hormone independence. Breast Cancer Res Treat 121: 293-300, 2010.

25. Klionsky DJ, Abeliovich H, Agostinis P, et al: Guidelines for the use and interpretation of assays for monitoring autophagy in higher eukaryotes. Autophagy 4: 151-175, 2008.

26. Seglen PO and Gordon PB: 3-Methyladenine: specific inhibitor of autophagic/lysosomal protein degradation in isolated rat hepatocytes. Proc Natl Acad Sci USA 79: 1889-1892, 1982.

27. Sanchez CG, Penfornis P, Oskowitz AZ, et al: Activation of autophagy in mesenchymal stem cells provides tumor stromal support. Carcinogenesis 32: 964-972, 2011.

28. Degenhardt K, Mathew R, Beaudoin B, et al: Autophagy promotes tumor cell survival and restricts necrosis, inflammation, and tumorigenesis. Cancer Cell 10: 51-64, 2006.

29. Karantza-Wadsworth V and White E: Role of autophagy in breast cancer. Autophagy 3: 610-613, 2007.

30. Lum JJ, Bauer DE, Kong M, et al: Growth factor regulation of autophagy and cell survival in the absence of apoptosis. Cell 120: 237-248, 2005 .

31. Karantza-Wadsworth V, Patel S, Kravchuk O, et al: Autophagy mitigates metabolic stress and genome damage in mammary tumorigenesis. Genes Dev 21: 1621-1635, 2007.

32. Boya P, Gonzalez-Polo RA, Casares N, et al: Inhibition of macroautophagy triggers apoptosis. Mol Cell Biol 25: 1025-1040, 2005.
33. Roorda BD, ter Elst A, Kamps WA and de Bont ES: Bone marrow-derived cells and tumor growth: contribution of bone marrow-derived cells to tumor micro-environments with special focus on mesenchymal stem cells. Crit Rev Oncol Hematol 69: 187-198, 2009.

34. Tian LL, Yue W, Zhu F, Li S and Li W: Human mesenchymal stem cells play a dual role on tumor cell growth in vitro and in vivo. J Cell Physiol 226: 1860-1867, 2011.

35. Sinha S and Levine B: The autophagy effector Beclin 1: a novel BH3-only protein. Oncogene 27 (Suppl 1): S137-S148, 2008.

36. Maiuri MC, Criollo A, Tasdemir E, et al: $\mathrm{BH} 3$-only proteins and $\mathrm{BH} 3$ mimetics induce autophagy by competitively disrupting the interaction between Beclin 1 and $\mathrm{Bcl}-2 / \mathrm{Bcl}-\mathrm{X}(\mathrm{L})$. Autophagy 3: 374-376, 2007.

37. Pattingre S, Tassa A, Qu X, et al: Bcl-2 antiapoptotic proteins inhibit Beclin 1-dependent autophagy. Cell 122: 927-939, 2005.

38. Chang NC, Nguyen M, Germain M and Shore GC: Antagonism of Beclin 1-dependent autophagy by BCL-2 at the endoplasmic reticulum requires NAF-1. EMBO J 29: 606-618, 2010.

39. Lian J, Wu X, He F, et al: A natural $\mathrm{BH} 3$ mimetic induces autophagy in apoptosis-resistant prostate cancer via modulating Bcl-2-Beclin1 interaction at endoplasmic reticulum. Cell Death Differ 18: 60-71, 2011. 